\title{
Egocentric spatial framework effects from single and multiple points of view
}

\author{
RUTH H. MAKI and MICHELLE N. MAREK \\ North Dakota State University, Fargo, North Dakota
}

\begin{abstract}
In three experiments, we tested the one-place, one-perspective rule formulated by Franklin, Tversky, and Coon (1992). This rule proposes that subjects take a neutral, external perspective when they must use multiple viewpoints to make decisions about the locations of objects in memorized scenes. We compared responding from a single viewpoint with responding from two viewpoints. In Experiments 1 and 2, we used a sentence verification procedure, and in Experiment 3, we compared a true-false verification procedure with a six-alternative forced-choice procedure. Under these various conditions, we observed egocentric spatial framework effects in that above-below judgments were faster than front-back judgments and front-back judgments were faster than right-left judgments. When responding from two points of view in a single place, our subjects took multiple intrinsic perspectives rather than one neutral external perspective as proposed by the one-place, one-perspective rule.
\end{abstract}

A consequence of humans' mobility is that the relative locations of objects in the space surrounding them changes as they change positions. People can use any of several possible frameworks to locate objects in space (see, e.g., Carlson-Radvansky \& Irwin, 1994; Taylor \& Tversky, 1996). In viewer-centered, or egocentric, frameworks, the locations of objects are defined with respect to the viewer's perspective as if the viewer is in the scene. Similarly, intrinsic reference frames can be used with respect to an object or another person in the scene. Other alternative frameworks are also possible. Locations can be mapped to an external frame of reference so that objects' locations are defined with respect to an environmentcentered reference frame. Descriptions of spatial relations depend on the spatial framework that is being used.

Use of an egocentric frame of reference is common, although individuals who are communicating will often use their listeners' frame of reference (an intrinsic frame) rather than their own (Schober, 1993, 1995). Franklin and Tversky (1990) asked college students to memorize scenarios in which an observer was in an environment with six surrounding objects. When indicating the locations of objects relative to the observer, the students took longest to respond to objects to the right and left of the observer, an intermediate time to respond to objects in front and back of the observer, and the shortest time to respond to objects above and below the observer. This

Portions of this paper were presented at the meetings of the Psychonomic Society in Los Angeles, November 1995. Our thanks to Nancy Franklin, who kindly supplied the scenarios that we used in these experiments and who suggested Experiment 2. Thanks also to Laura Jacobson, Paul Enderson, and Michelle Schumacher, who tested some of the subjects. This paper was written while the first author was a Visiting Professor at Michigan State University. The hospitality and lively discussions of the Cognitive Interest Group are appreciated. Correspondence should be addressed to R. H. Maki, Department of Psychology, Texas Tech University, Lubbock, TX 79409-2051 (e-mail: rumaki@ttu.edu). pattern was interpreted as showing the use of an egocentric frame of reference for verification of the locations of objects in imagined environments. Franklin and Tversky hypothesized that the difficulty of identifying different locations depends on how constant the locations are in the environment as one moves and on how asymmetrical the locations are around one's body. Although the exact locations of objects in the vertical dimension change as one moves through the environment, the vertical location relative to the observer (above and below) stays fairly constant. Furthermore, the vertical dimension is correlated with gravity, and individuals are asymmetrical at their heads versus their feet. Thus, it should be easy to identify locations in the vertical dimension. The two horizontal dimensions change with movement, so they should be more difficult. The front-back dimension has the advantage of being correlated with the direction of normal movement and with the asymmetry of the front and back of one's body, but the right-left dimension is not correlated with the normal route of movement and is based on relatively symmetrical right and left body halves. This analysis of an egocentric spatial framework suggests that individuals should be able to identify the locations of objects in the above-below dimension the easiest, followed by the front-back dimension, with the right-left dimension being the mọst difficult. Hintzman, O'Dell, and Arndt (1981) have presented partial support for an egocentric spatial framework in that they found that college students could identify memorized objects in locations to the front faster than objects in locations to the back, which in turn were identified faster than objects to the right and left. Franklin and Tversky (1990) extended support for the use of an egocentric spatial framework in that they found that right-left took longer than front-back, which took longer than above-below, when college students identified the locations of imagined objects around themselves as they stood in imagined environments. 
The spatial framework analysis is supported by the order in which children learn the dimensions. Clark (1980) showed that children can identify the top and bottom of objects at a younger age than that at which they can identify the front and back. Harris (1972) showed that children are able to discriminate front and back earlier than right and left. There is a large literature showing that right-left discriminations are more difficult than above-below discriminations. Rudell and Teuber (1963) showed that many children below age 6 could not learn to discriminate a right-facing $U$ shape from a left-facing U shape. Harris (1975) found that drawing a circle to the right or left of a central point took longer than drawing one above or below that point. Maki, Maki, and Marsh (1977) showed that college students took much longer to verify the locations of two horizontally oriented symbols as opposed to those of two vertically oriented symbols and that they took longer to verify the locations of states of the United States that had an east-west relationship as opposed to those that had a north-south relationship. Logan (1995) showed that when the shifting of attention was guided by the words right and left, responding to the location of a target took longer than when attention was guided by above and below.

The difficulty with right and left is pervasive, but in a few instances in the literature, right and left have not been more difficult than above and below. Maki, Grandy, and Hauge (1979) found that upward and downward pointing arrows could be identified as fast as rightward and leftward pointing arrows if the concepts of right and left were bypassed by having subjects learn new singleletter labels for the different arrows. They concluded that right and left are difficult for college students whenever the spatial terms, and hence the conceptual representations of right and left, must be accessed in a task. Two more recent studies support this analysis. Sholl and Egeth (1981) found that the right-left difficulty disappeared in a mirror image discrimination task when the verbal labels right and left were not involved in the task. When Logan (1995) replaced the labels above, below, right , and left with sequentially ordered digit cues, subjects shifted their attention to right and left locations as quickly as to above and below locations.

Franklin, Tversky, and Coon (1992) found some conditions in which the right-left difficulty was either very small or nonexistent. College students memorized scenarios containing six objects and two characters having different viewpoints. They were then asked to identify which of six objects was in a specific location relative to either one or the other character. This procedure produced several instances in which right-left decisions did not take significantly longer than above-below decisions. In addition, they found that the egocentric spatial framework effects that Franklin and Tversky (1990) reported (i.e., above-below faster than front-back and front-back faster than right-left) were not significant under several of their conditions. In their Experiment 1, two characters in a scenario were surrounded by a single set of objects. On a random half of the trials, subjects re- sponded from the viewpoint of self in the scenario, and on the other half of the trials, they responded from the viewpoint of the other person in the scenario. Franklin and Tversky did not find reliable differences between front-back and above-below in either condition, and right-left was not reliably different from front-back when the self viewpoint was queried. The absence of a right-left difficulty is surprising, given that the words right and left were used in the task. In addition, the mean for right-left was actually a bit faster than the mean for above-below. Franklin et al. (1992) also showed that the spatial framework effects were not significant in other situations in which multiple viewpoints were needed for one to respond to locations in a scenario. These included a situation in which two characters were in different parts of the same environment, each surrounded by six different objects; a situation in which one viewer overlooked two different locations, each of which contained a different character with six different surrounding objects; and a single person oriented in different ways among six objects at different times. In each of these conditions, above-below, front--back, and right-left produced response times that were not different enough to be significant. However, in many of the conditions, the means for right-left were longer than the means for above-below and front-back.

Franklin et al. (1992) interpreted their results as suggesting that subjects took an external, environmentally based perspective that was not egocentric for the self character or intrinsic to the other character. Thus, subjects were able to take both characters' viewpoints without computing the specific spatial relations with respect to each character. Franklin et al. suggested a one-place, one-perspective rule to incorporate the idea that subjects used a neutral, external perspective that included all spatial relations from both characters' viewpoints. Taking an external perspective when two characters are in a single scene may serve to reduce the mental effort that would be required in order to take two different individuals' viewpoints. Schober and Bloom (1995) showed that it is particularly difficult to take the viewpoint of another individual who is facing a different way in the same environment as one's own.

The implication of the one-place, one-perspective rule is that responding to multiple viewpoints by taking an external perspective makes the spatial relations equally available. Although Franklin et al.'s (1992) results provided evidence for such equiavailability, they did not include a condition in which subjects responded from only one viewpoint, so they could not make a comparison with times showing egocentric responding. Responding from an external, as opposed to an egocentric, perspective may have increased the time to respond to above-below relative to front-back and right-left, or it may have decreased the time to respond to front-back and right-left relative to above-below.

Other data suggest that scenes in which multiple perspectives are possible result in interference and make spatial judgments difficult. Carlson-Radvansky and Irwin 
(1994) had subjects make decisions about whether or not one object was above another object. Decisions were fastest when viewer-centered (egocentric), environmentcentered (external), and object-centered (intrinsic) axes were congruent. When the referent object was turned so that its top was not canonical with the environmental and egocentric up, viewers took a long time both to accept and to reject the statements that a target object was above the noncanonical reference object. Carlson-Radvansky and Irwin suggested that multiple frames of reference were activated and that they competed, making spatial decisions difficult. In the Franklin et al. (1992) situation, two frames of reference (an egocentric one for the self and an intrinsic one for the other person) could be activated when the two individuals faced in different directions. This should make the spatial location task difficult, and that difficulty should be greater for the dimensions that change as the individuals turn-namely, for the front-back and right-left dimensions as compared with the more stable above-below dimension. In contrast, Franklin et al. concluded that only a single, external frame of reference was activated and that this made the spatial locations equally available.

Further support for the idea of interference and increased difficulty with multiple frameworks comes from two recent studies. Schober (1993) found that speakers who were communicating the location of an object to another individual were fastest when both individuals shared the same viewpoint, and slowest when the addressee was facing the communicator. De Vega (1994) conducted a study that was similar to Franklin et al.'s (1992) studies, except that the scenarios were simplified. The viewpoints of two characters in a scene were the same or different, and either one or both of the individuals were involved in specific test items. De Vega investigated front-back versus right-left relations. He found that right--left statements took longer to verify than frontback statements in all situations. Items involving two characters took longer than items involving a single character, and items involving characters taking two different viewpoints took longer than items with single viewpoints. De Vega concluded that subjects constructed models in memory from a neutral, external perspective that included all characters and objects in a scene. Then, during verification trials, they computed the specific spatial relations with respect to the individual involved in the verification item. Thus, the perspective from which subjects responded was intrinsic with respect to the specific character involved in the question. Computing the intrinsic right-left spatial relations took longer than computing the frontback spatial relations.

De Vega's (1994) conclusion that subjects used a perspective-free external model is similar to Franklin et al.'s (1992) interpretation of their data. However, de Vega argued that an egocentric-type framework was then used to compute the specific spatial relations with respect to the character named in the verification item. This produced longer times for right-left than for front-back. In contrast, Franklin et al. found very small or no dimension effects in their studies.

The use of an external as opposed to egocentric and other character-intrinsic perspectives also has implications for within-dimension effects. The spatial framework analysis (Franklin \& Tversky, 1990) predicts that front should be responded to more quickly than back. This is because front is primarily associated with navigation, and an individual can see what is in front but must make some transform to see what is in back. Both Franklin and Tversky and Hintzman et al. (1981) found evidence that front was responded to faster than back when their subjects judged the locations of objects with respect to an observer in memorized scenes. Bryant, Tversky, and Franklin (1992) found that the difference between front and back occurred only when the observer was taking an egocentric viewpoint. If the observer took an external viewpoint looking in at the scene, fronts and back were equivalent. The hypothesis that front is easier than back only when one takes an egocentric or intrinsic viewpoint is supported by studies in which children identified the fronts and backs of various types of objects. Neither Clark (1980) nor Harris and Strommen (1979) found that children identified the fronts of objects more easily than the backs, presumably because the children were using external and not egocentric reference frameworks.

The present experiments were designed to determine whether egocentric and intrinsic spatial framework effects occur when subjects imagine scenarios involving multiple perspectives. We used the materials from Franklin et al.'s (1992) first experiment, which showed no dimension effects when the self viewpoint was queried. In Experiments 1 and 2, we used verification sentences of the type used by de Vega (1994) to determine whether this type of query might encourage the use of egocentric spatial frameworks with Franklin et al.'s scenarios. We used all three dimensions: above-below, front-back, and right-left. De Vega found that right-left took longer than front-back, but he did not investigate the other comparison that is critical in Franklin and Tversky's (1990) spatial framework model-namely, front-back versus above-below. In Experiment 3, we used single-word viewpoint and location queries that were the same as those used by Franklin et al. In all three experiments, we compared responding from two viewpoints with responding from a single viewpoint in order to examine the size of the spatial framework effects under different spatial processing demands.

\section{EXPERIMENTS 1-2}

In both Experiments 1 and 2, subjects learned scenarios that contained two characters who sometimes shared the same viewpoint, but who usually took different viewpoints in imagined environments. One of these viewpoints was that of the self, so use of an egocentric framework was possible. After learning each scenario, college students verified the spatial relations between the six ob- 
jects in the scenario and either one or both of the characters. In the self-viewpoint, single-perspective condition, all verifications were made with respect to self. In the other-viewpoint, single-perspective condition, all verifications were made with respect to the other person in the scenario. In the multiple-perspective condition, a random half of the verification trials were from the viewpoint of self and a random half were made from the viewpoint of the other character. De Vega (1994) found that right-left took longer than front-back both when items involved two characters and when they involved a single character. Franklin et al. (1992) found either no dimension effects or very small right-left versus front-back effects in their studies, in which both viewpoints were queried in a random order. We examined the front-back versus above-below and right-left versus front-back differences, because those are critical for showing that subjects are using an egocentric spatial framework. We also analyzed the right-left versus above-below difference, because that is what has been used in previous studies showing the right-left difficulty. The main question was whether these effects would be smaller in the multipleperspective condition (because a neutral perspective with equally available spatial relations was used) than in the single-perspective conditions (because the egocentric perspective of the tested character was used).

Experiments 1 and 2 differed in whether or not advance information about the perspective taken in the verification question was given. Franklin et al. (1992) gave advance viewpoint information, but their advance information was a necessary part of the trial. Without it, their subjects would not have known whether to respond from the viewpoint of self or other. In our procedure, that viewpoint information was redundant in that the viewpoint was contained in each verification sentence, as well as in the advance information. Having viewpoint information in our Experiment 1 in advance may have made our subjects likely to respond from specific egocentric viewpoints rather than from a single, external viewpoint as reported by Franklin et al. This was tested in Experiment 2, which was identical to Experiment 1, except that no advance viewpoint information was presented.

\section{Method}

Subjects. A total of 48 volunteers from introductory psychology classes at North Dakota State University participated in Experiment 1 for extra credit. Sixteen were randomly assigned to each of three groups: the single-perspective, self-viewpoint group; the single-perspective, other-viewpoint group; and the multipleperspective, mixed-viewpoint group. Four subjects who made more than $25 \%$ errors in this two-alternative forced-choice task were eliminated. One was eliminated from the single, self condition; two were eliminated from the single, other condition; and one was eliminated from the multiple-perspective condition.

Another group of 48 volunteers from lower level psychology courses at North Dakota State University participated in Experiment 2 . They were randomly assigned to three groups of 16 subjects each: the single-perspective, self condition; the single-perspective, other condition; and the multiple-perspective, mixed condition. The data from all subjects were used, because no one made more than $25 \%$ errors.
Materials. The narratives were the ones used by Franklin et al. (1992) in their Experiment 1. The practice narrative was identical to theirs, and four of their six experimental narratives were used. The first part of each narrative described two observers. One observer was given the title of self, and the other was given a common name. Both observers were standing in the same scene, surrounded by the same objects, but usually facing in different directions. The site of the practice narrative was in a workshed, and the sites of the four experimental narratives were a construction site, a navy ship, a space museum, and a hotel. Six different objects surrounded the two individuals in each narrative. The direction of each object was always given first with respect to the viewpoint of self. After all objects had been thus located, their locations were described with respect to the other person. The navy ship narrative is given below as an example:

You are in the United States Navy, on the USS Tugmaster. At the moment, you are standing on the upper deck with Hank, another sailor. You and Hank are on watch duty together and are standing at the edge of the observation deck overlooking the deck below. Beyond your right is a cannon. It is mounted to the deck by a series of heavy bolts. Beyond your feet is an antenna. It is shaped into a concave dish. Beyond your front is an anchor. It appears to have been corroded by many years of exposure to salt water. Beyond your left is an oversized bell. A knotted, yellow cord hangs from the inside. Beyond your back is Hank. Beyond your head is a lifeboat. It hangs by two ropes from a set of pulleys. Beyond Hank's front is you. Beyond Hank's right is the cannon. Beyond Hank's left is the bell. Beyond Hank's feet is the antenna. Beyond Hank's back is an American flag. Its thin material has gotten wet from the sea spray. Beyond Hank's head is the lifeboat.

The second part of each narrative was written in three blocks Each of the blocks began with sentences describing reorientations of the observers. The other observer was always reoriented first. The observers never faced each other. These reorientation sentences were followed by 12 sets consisting of 2 filler sentences and 1 verification sentence. The 2 filler sentences described a detail of the object in front of "self," although the object was not named. The 3 rd sentence was the verification sentence, which described the location of an object with respect to either the self or the other person. In a block, there were 12 different verification sentences, 1 true sentence, and 1 false sentence for each of the six objects. An example of the 3 reorientation sentences, 2 filler sentences, viewpoint information, and a verification sentence is given below for the selfviewpoint, left, true condition. The verification sentence is printed in bold, and the subjects' expected responses are in brackets.

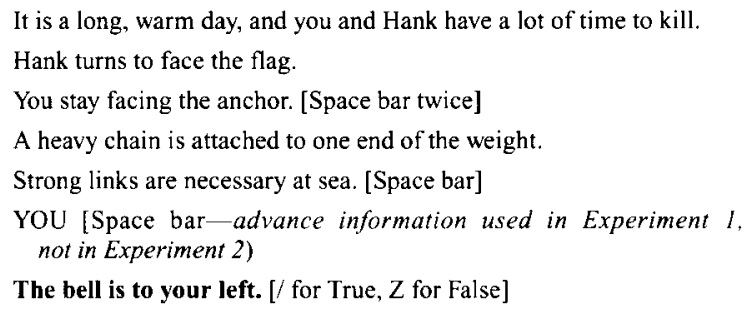

Procedure. Each narrative was presented on a computer monitor. The narrative began with a title that contained the location of the scene and the name of the second observer. Next, a list of the six objects that would be included in the narrative was presented. Then a story, which described each of the objects with respect to their locations relative to the two observers' locations (right/left, front/back, or above/below), was presented on the monitor. The subjects were allowed to study the title, the objects, and the story for as long as they needed in order to be confident about the locations of the objects. They pressed the keyboard's space bar when they understood the objects' locations with respect to the observers. The narrative was erased and the subjects were asked whether they were sure that they knew the objects' locations with respect to each observer. If they responded "yes," they proceeded to the next part. If they responded "no," the narrative was presented for study again. 
The subjects were then given the reorientation information where one or both of the observers turned to face a new object. Then the two sentences of filler text were presented on the monitor until the subject indicated understanding by pressing the space bar. In Experiment 1 , these were followed by viewpoint information, with either the word "YOU" or the name of the other person presented on the monitor. The subjects were instructed to press the space bar when they had understood the viewpoint, and to get ready to respond by placing their left and right index fingers on the "/" and the "Z" keys. In Experiment 2, the subjects were simply instructed to place their fingers on the "/" and " $Z$ " keys and get ready to respond. Next, the verification sentence was presented and the subjects pressed the "/" key if it was true and the " $Z$ " key if it was false.

After the response to the sentence, another screen of two lines of filler text was presented, followed by viewpoint information (in Experiment 1) and another verification sentence. After a block of 12 verification sentences, the observers reoriented themselves and the subject continued with a new block of verification sentences. There were three orientation switches for each narrative, there was one practice narrative, and there were four experimental narratives.

During practice, the subjects were given feedback after each trial If their response was correct, the word "correct" was displayed on the screen for $1 \mathrm{sec}$. If the response was incorrect, a tone beeped for $500 \mathrm{msec}$ and the word "incorrect" was flashed on the screen for $1 \mathrm{sec}$. After a subject made three errors during a block, the message "You have made X errors. Please go more slowly and try to be more accurate" was presented on the screen for $10 \mathrm{sec}$. This message was repeated with the current number of errors replacing the $\mathrm{X}$ following all error trials for that block. After the practice narrative was completed, the four experimental narratives were presented and tested. No feedback was given for the experimental narratives.

Design. All subjects responded to the same practice narrative and to the same four critical narratives, but the order in which the critical narratives was presented was randomized across subjects so that each narrative was presented in each temporal order about equally often. For Experiment 1, in the single-perspective conditions, the viewpoint information was always the same: YOU in the self-viewpoint condition, and the other person's name in the otherviewpoint condition. In the multiple-perspective condition, the advance viewpoint information reliably indicated the viewpoint from which the forthcoming sentence would be written. In both Experiments 1 and 2 in the single-perspective conditions, each object was included in a true and in a false sentence in each block. In the multiple-perspective condition, the locations of all six objects were verified in each block from the viewpoint of self and from the viewpoint of the other person. Half were true, and half were false. The use of specific objects in true and false sentences for each observer was balanced across subjects so that all objects were tested equally often from each viewpoint for true and for false sentences across pairs of subjects. True and false queries concerning specific objects in specific locations were presented randomly in each block.

\section{Results}

Advance information about the viewpoint to be used on each trial was provided in Experiment 1, but it was not provided in Experiment 2. Otherwise, these two experiments were identical, although they were conducted during different semesters and the subjects were not randomly assigned to experiments. In order to simplify the description of the analyses, the data from Experiments I and 2 were combined for analysis of the reaction times (RTs) to verify each sentence and for analysis of errors made in verifying the truth of the sentences. Separate analys $r$ were conducted for the self- and the otherviewpoint conditions. Each analysis of variance (ANOVA) was a 2 (experiment/advance information) $\times 2$ (number of perspectives) $\times 3$ (spatial dimension) $\times 2$ (truth) mixed-design analysis. For each analysis, experiment (advance information) and number of perspectives were between-subjects variables. The data in the singleperspective conditions were from either the self or the other group, and the data in the multiple-perspective condition were from the half of the trials referring to self in the analysis of the self-viewpoint and to other in the other-viewpoint analysis.

Significant dimension effects were followed up by three pairwise comparisons. The above-below RT was compared with the front-back RT and the front-back RT was compared with the right-left RT because these are the main comparisons to show the use of an egocentric spatial framework. The right-left RT was compared with the above-below RT in order to investigate the right-left difficulty. The significance of each pairwise comparison was assessed both with the Tukey honestly significant difference (HSD) correction and with no correction, using the least significant difference (LSD). As suggested by Keppel and Zedeck (1989), if a difference was significant with the HSD correction, it was considered to be unlikely to have occurred by chance. If it was not significant with the LSD, it was considered to have probably occurred by chance. We will suspend judgment about any difference that was significant without the correction, but that did not allow rejection of the null hypothesis with it.

Self viewpoint. Mean RTs from the viewpoint of self are shown in Figure 1. Overall, RTs were faster in Experiment 2, without advance information, than in Experiment 1 , with advance information, although this effect may have been due to a different sample of subjects and may not have been a real effect of advance information. True responses were faster than false responses, and RTs differed across dimensions, with right-left taking longer than front-back and front-back taking longer than above-below. Each of these effects produced a significant main effect at $p<.05$, the level of significance to be used for all analyses $\left[F(1,58)=4.69, M S_{\mathrm{e}}=2,911,830\right.$, for the experiment/advance information effect; $F(1,58)=$ $14.91, M S_{\mathrm{e}}=319,640$, for truth; and $F(2,116)=40.57$, $M S_{\mathrm{e}}=415,367$, for spatial dimension].

Figure 1 also shows the Tukey HSD range for each specific comparison. The Tukey critical difference is shown on the graph between the bars representing above-below and front-back and between bars representing front-back and right-left. The Tukey HSD for above-below versus right-left is shown to the right of the right-left bars. Differences are significant if the data bars fall outside of the indicated critical range. As can be seen in Figure 1, RTs generally increased from above-below to front-back to right-left. Overall, the difference in RTs for above-below as compared with front-back was larger in the multipleperspective condition than in the single-perspective condition. Although the overall perspective $\times$ dimension interaction was not significant $\left[F(2,116)=2.22, M S_{\mathrm{e}}=\right.$ $415,367]$, there was a significant interaction between di- 


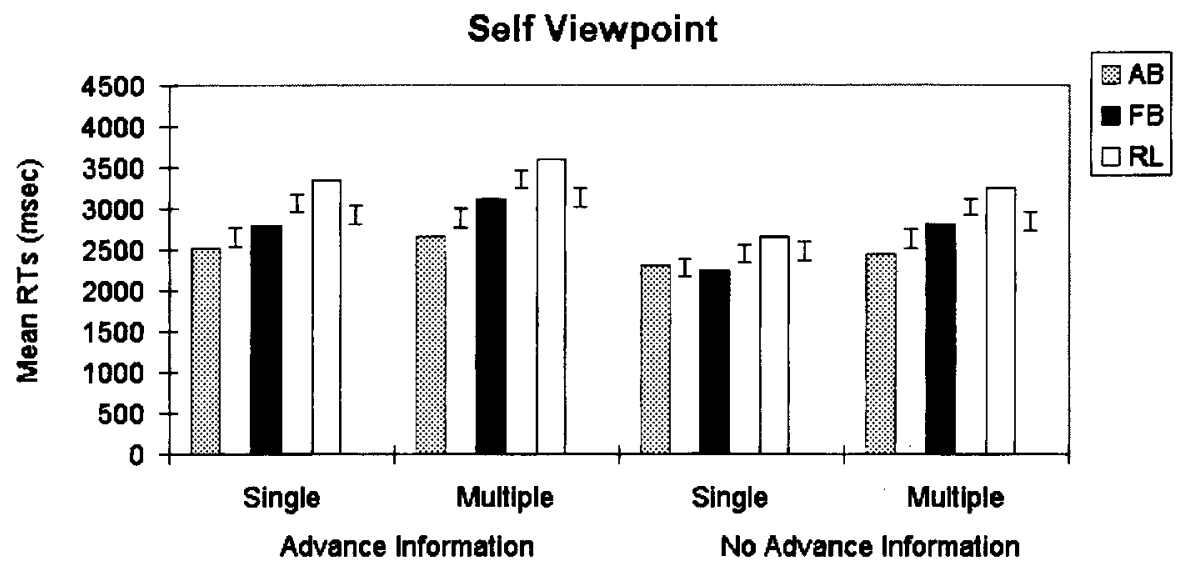

\begin{abstract}
Figure 1. Mean reaction time (RTs) in milliseconds for above-below (AB), front-back (FB), and right-left (RL) locations in the self-viewpoint, single- and multiple-perspective conditions in Experiment 1, with advance information, and Experiment 2, without advance information. Error bars are centered on the means of surrounding data bars to show the Tukey HSDs for above-below versus front-back and front-back versus right-left. The HSD for right-left versus above-below is to the right of the right-left bar. Data bars that are higher and lower than the error bars show significant differences.
\end{abstract}

mension and number of perspectives when only the above-below and front-back dimensions were considered $\left[F(1,58)=5.94, M S_{\mathrm{e}}=251,907\right]$. When above-below was compared with front-back separately for the singleand multiple-perspective conditions, above-below was reliably faster than front back for the multiple-perspective condition, but not reliably faster for the single-perspective condition.

Proportions correct were analyzed to determine whether the effects observed in Experiments 1 and 2 might be due to a speed-accuracy tradeoff. Overall, the proportion correct in the self condition was .87. True responses produced higher accuracy than did false responses [.89 vs. $\left..86 ; F(1,58)=6.13, M S_{\mathrm{e}}=.013\right]$. Accuracy differed for above-below, front-back, and right-left $[.90, .86$, and $\left..87 ; F(2,116)=9.95, M S_{\mathrm{e}}=.012\right]$. This pattern was the same for single and multiple perspectives $(F<1$ for the perspective $X$ dimension interaction), but the pattern of accuracy across dimensions differed for Experiments 1 and $2\left[F(2,116)=4.66, M S_{\mathrm{e}}=.012\right]$. For Experiment 1, with advance information, correct responding showed the same pattern as did RTs, with proportions correct of .91 for above-below, .85 for front-back, and .81 for right-left $\left[F(2,56)=17.35, M S_{\mathrm{e}}=.009\right]$. Pairwise comparisons with Tukey HSD corrections showed that above-below decisions were more accurate than the front-back decisions, and front-back decisions were more accurate than right-left decisions. In contrast, in Experiment 2, with no advance information, accuracy did not differ reliably $\left[F(2,60)=1.18, M S_{\mathrm{e}}=.016\right]$ for above-below (.89), front-back (.87), or right-left (.90).

Other viewpoint. Mean RTs from the viewpoint of the other character in each scenario are shown in Figure 2. Overall, above-below decisions were faster than front-back and right-left decisions, and front-back de- cisions were faster than right-left decisions $[F(2,114)=$ $78.80, M S_{\mathrm{e}}=515,302$ for the dimension main effect]. This pattern did not differ with or without advance information or for single or multiple perspectives $[F(1,57)=$ $1.40, M S_{\mathrm{e}}=515,302$, and $F<1$, respectively]. True responses were faster than false responses $[F(1,57)=23.78$, $\left.M S_{\mathrm{e}}=318,657\right]$, and truth interacted with dimension $\left[F(2,114)=3.60, M S_{\mathrm{e}}=299,059\right]$. For true responses, mean RTs for above-below, front-back, and right-left were $2,736,3,050$, and $3,747 \mathrm{msec}$, respectively. For false responses, the mean RTs were $2,812,3,484$, and $4,100 \mathrm{msec}$. The dimension effects were present for both true and false responses, but they were larger for false responses $\left[F(2,114)=46.23, M S_{\mathrm{e}}=351,317\right.$, for true; and $F(2,114)=54.34, M S_{\mathrm{e}}=463,044$, for false]. Each pairwise comparison was significant for true and false responses with Tukey HSD corrections.

The overall proportion correct in the other-viewpoint condition was .86. Proportion correct was higher in Experiment 2, without advance information (.89), than in Experiment 1 , with advance information $[.82 ; F(1,57)=$ $\left.9.12, M S_{\mathrm{e}}=.060\right]$. When all responses were made from the perspective of the other person, proportions correct to above-below, front-back, and right-left were $.92, .86$, and .78. When half of the responses were made from the perspective of self and half from the perspective of the other person, proportions correct for responses from the other characters' viewpoint were $.88, .87$, and .84 , for above-below, front-back, and right-left decisions. The ANOVA showed a main effect of dimension $[F(2,114)=$ $\left.12.55, M S_{\mathrm{e}}=.012\right]$ and an interaction between dimension and number of perspectives $\left[F(2,114)=3.86, M S_{\mathrm{e}}=\right.$ $.012]$. The dimension effect was significant for the pure other condition $\left[F(2,56)=18.88, M S_{\mathrm{e}}=.009\right]$. Pairwise comparisons corrected with the Tukey HSD showed that 


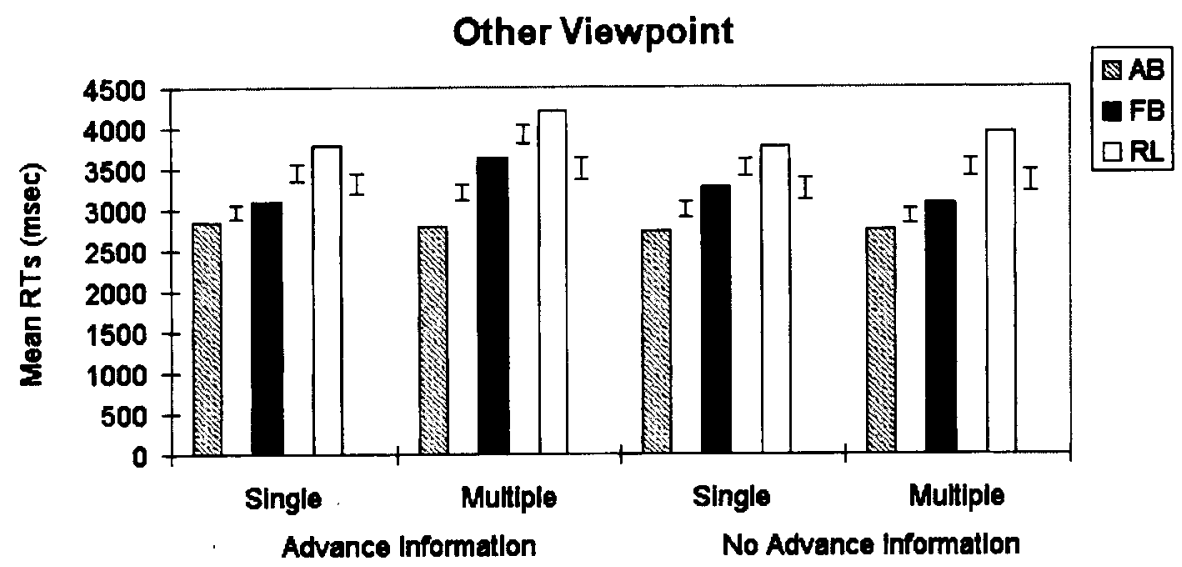

Figure 2. Mean reaction time (RTs) in milliseconds for above-below (AB), front-back (FB), and right-left (RL) locations in the other-viewpoint, single- and multiple-perspective conditions in Experiment 1, with advance information, and Experiment 2, without advance information. Error bars are centered on the means of surrounding data bars to show the Tukey HSDs for above-below versus front-back and front-back versus right-left. The HSD for right-left versus above-below is to the right of the right-left bar. Data bars that are higher and lower than the error bars show significant differences.

above-below decisions were more accurate than both front-back and right-left decisions, and front-back decisions were more accurate than right-left decisions. When subjects responded from multiple perspectives, however, there was not a significant difference in accuracy as a function of dimension $\left[F(2,58)=1.05, M S_{\mathrm{e}}=.014\right]$.

Mixed-viewpoint condition. Franklin et al. (1992) did not test the single-viewpoint conditions, so all of their analyses were conducted as within-subjects ANOVAs on data from a multiple-perspective condition. We also analyzed the multiple-perspective, mixed-viewpoint condition so that we could look for differences between self and other as the reference character. The data for this analysis are shown in the multiple-perspective conditions for the self viewpoint in Figure 1 and for the other viewpoint in Figure 2. Overall, responding from the other viewpoint took longer than responding from the self viewpoint $\left[3,392\right.$ vs. $2,973 \mathrm{msec} ; F(1,29)=47.44, M S_{\mathrm{e}}=$ $343,732]$. RTs to above-below, front-back, and right-left from the viewpoint of self were $2,548,2,955$, and $3,417 \mathrm{msec}$, respectively. From the viewpoint of the other character, the corresponding RTs were $2,766,3,344$, and $4,067 \mathrm{msec}$. RTs to the three dimensions differed $\left[F(2,58)=43.39, M S_{\mathrm{e}}=848,344\right]$, but the dimension differences were larger from the other viewpoint than from the self viewpoint $\left[F(2,58)=5.82, M S_{\mathrm{e}}=252,090\right]$. All pairwise comparisons were significant with Tukey HSD corrections, but the differences between front-back and right-left and between above-below and right-left were larger from the other than from the self viewpoint.

Within-dimension effects. If subjects were responding from egocentric and intrinsic spatial frameworks by taking the viewpoint of self and other in turn, front should be faster than back because front is perceptually salient from the intrinsic viewpoint of a person. We ex- amined this effect in the combined data from Experiments 1 and 2. When sentences were verified from the viewpoint of self, front was faster than back $(2,665 \mathrm{vs}$. $2,934 \mathrm{msec}$ ), and from the viewpoint of other, front was also faster than back $(3,188$ vs. $3,426 \mathrm{msec})$. The ANOVA showed that each of these effects was significant $\left[F(1,58)=4.32, M S_{\mathrm{e}}=1,040,677\right.$, and $F(1,57)=4.15$, $M S_{\mathrm{e}}=837,771$, for self and other, respectively]. Neither of these effects interacted with number of perspectives $\left[F \mathrm{~s}(1,58) \leq 2.41, M S_{\mathrm{e}}=1,040,677\right]$ or with advance information $\left[F \mathrm{~s}(1,57) \leq 1.52, M S_{\mathrm{e}}=837,771\right]$.

\section{Discussion}

There was evidence for egocentric spatial framework effects in both the single- and multiple-perspective conditions, whether subjects were responding from the viewpoint of self or the other person in the scenario. Front-back took longer than above-below in all conditions except the single-perspective, self condition. Right-left took longer than front-back and above-below in all conditions. In contrast to Franklin et al.'s (1992) finding, we did not find that taking multiple perspectives reduced egocentric spatial framework responding. If anything, we found more consistent evidence for spatial framework effects in the multiple-perspective condition than in the single-perspective conditions.

Interestingly, knowing the viewpoint in advance had very few effects. It was expected that subjects who were responding from both the other and the self viewpoints across trials would benefit from the advance information, but they did not. Advance information tended to slow down subjects in the self condition, probably because they were set to respond from the self viewpoint anyway. It did take students in the multiple-perspective condition longer to respond to other than to self, and the spatial 
framework effects involving right-left were larger when the viewpoint was that of the other person. Franklin et al. (1992) also found that self was faster than other when an equal number of questions was given for each character. They concluded that subjects had foregrounded self and could respond fastest from that viewpoint. It is possible that this type of foregrounding resulted in faster times and also made objects in the difficult right-left locations more accessible. However, it is also possible that the object locations were learned better from the perspective of self because the locations from the self perspective were always learned before the locations from the other character's perspective. In spite of these differences between self and other, strong spatial framework effects were observed from both perspectives, suggesting that subjects took the intrinsic perspective of each character as needed for each trial.

Further support for responding from egocentric or intrinsic spatial frameworks came from the finding that front was faster than back. According to Bryant et al. (1992), this pattern occurs when subjects respond from an egocentric or intrinsic framework. The fact that this occurred from both the self and the other viewpoint supports the idea of responding in an "egocentric" fashion from the viewpoint of whichever character was in the verification sentence. However, the front-back effects did not interact with number of perspectives. Thus, there was no evidence for less egocentric or intrinsic responding when multiple as opposed to single viewpoints were queried.

\section{EXPERIMENT 3}

In Experiments 1 and 2, intrinsic spatial framework effects were observed in the multiple-perspective condition, whether responses were made from the viewpoint of self or the other character. The multiple-perspective, self-viewpoint condition produced no spatial framework effects in Franklin et al.'s (1992) Experiment 1. They found no difference between either front-back and above-below, or front-back and right-left. They did not explicitly test right-left and above-below, but the mean right-left RT was shorter than the mean above--below RT. Although we used the same scenarios as those of Franklin et al., there are several differences between our experiments and Franklin et al.'s experiments. A main difference is that we used sentence verification. Franklin et al. presented three separate screens of information: the viewpoint, the location term, and all six objects. They had subjects select the correct object from among the six. De Vega (1994) conducted an experiment that was similar to both ours and Franklin et al.'s, and he found that right-left took consistently longer than front-back. Like us, de Vega used sentence verification. It may be that the sentence verification procedure produces egocentric spatial framework effects, but that selecting from among all objects does not. In our experiments and in de Vega's, the incorrect object was always from the opposite pole of the tested dimension. This may encourage di- mensional encoding, which may result in egocentric spatial framework effects.

In Experiment 3, we tested subjects in a condition that replicated Franklin et al.'s (1992) Experiment 1. In addition, we compared a true-false procedure in which only same-dimension objects served as incorrect items on false trials with a procedure in which all objects were present as alternatives on each trial. For all subjects, viewpoint information (self or other) was given first, followed by the location (front, back, head, feet, right, or left). Half of the subjects then selected the correct object from among all six possibilities; the other half made true-false decisions when presented with either the correct object or the object from the opposite pole of the dimension. Within these two response-type groups, half of the subjects always responded from the viewpoint of self, and half responded from the viewpoint of self or other on randomly selected trials. We did not test a pure other condition in this experiment; subjects in the single-perspective condition always responded from the viewpoint of self.

To be consistent with the finding of Franklin et al. (1992), we expected to see smaller spatial framework effects in the multiple-perspective than in the singleperspective condition when all six objects were given as choices. If dimensional responding is encouraged by having incorrect objects from the opposite end of the dimension, when subjects made true-false decisions we expected to see spatial framework effects much like those of our Experiments 1 and 2.

\section{Method}

Subjects. Sixty-four subjects completed the experiment for extra credit in lower level psychology courses at North Dakota State University. Half were randomly assigned to true-false condition and half were assigned to the six-alternative forced-choice (6 AFC) condition. Within each of these conditions, half responded from the viewpoint of self on each trial and half responded from the viewpoint of self on a random half of the trials and from the viewpoint of the other character on the other half of the trials. Data from 3 subjects were dropped because of high error rates. Two subjects were dropped from the multiple-perspective, true-false condition because they made more than $25 \%$ errors (chance $=50 \%$ ). One subject was dropped from the multiple-perspective, $6 \mathrm{AFC}$ group because his responses were at chance, with about $17 \%$ correct.

Procedure. The narratives, reorientation, and filler sentences from Experiments 1 and 2 were used. However, instead of verification sentences being presented, the question was presented in 3 parts. First, the subjects were presented with the viewpoint from which they were to answer. Half of the subjects were always presented with the self viewpoint, and half were presented with the self viewpoint on half of the trials and the viewpoint of the other person on the remainder of the trials. After the subjects pressed the space bar to indicate that they understood the viewpoint, the viewpoint information was replaced on the screen by a direction from the designated observer ("head," "feet," "front," "back," "left," or "right"). The subjects were instructed to press the space bar as soon as they knew which object was in the designated location from the observer. The final part of the question consisted of a list of the objects surrounding the observer from which the subjects were to choose. In the $6 \mathrm{AFC}$ condition, all six objects were presented in a horizontal row on the screen with the numbers 1 through 6 beneath them. The order of the objects was randomized on each trial. The 
subjects were to indicate the correct object by pressing a number from 1 to 6 on the keypad on the right side of the keyboard. They were asked to use the three middle fingers of their right hands to do this. The other half of the subjects were presented with a true-false task in which the presented object was or was not the object in the designated location. If the object was incorrect, it was always from the opposite end of the correct dimension-for example, the object to the right on "left" trials. The subjects in the true-false condition were instructed to rest their index and fourth fingers on the numbers 1 and 3 on the keypad at the right of the keyboard and to press the 1 key for "true" responses and the 3 key for "false" responses. Thus, both groups rested the first three fingers of their right hands on three keys on the keypad on the computer keyboard. The subjects were urged to make all choices as quickly as possible, but also to be as accurate as possible. Furthermore, they were asked to study the location until they were sure which object was in the designated location.

\section{Results}

With the three-part question procedure (viewpoint, location word, response choice), times to encode the specific location words should differ as a function of dimension with right and left taking longer than front and back, which should take longer than head and feet (above and below). The subjects were instructed to decide which object was in the location during the time when the location was presented, so there should be no spillover effects of location into the choice time. However, Franklin et al. (1992) did find spillover effects, so they reported their results summed across location word time and choice time. We found no significant spillover effects for dimension in choice time for the 6 AFC condition. Mean choice RTs of 2,151, 2,289, and 2,324 msec for above-below, front-back, and right-left, respectively, were not significantly different $[F(2,58)=1.16$, $\left.M S_{\mathrm{e}}=219,513\right]$. In contrast, the times to study the location words were significantly different $[F(2,58)=23.80$, $\left.M S_{\mathrm{e}}=264,402\right]$ at $1,850 \mathrm{msec}$ for above-below, $2,088 \mathrm{msec}$ for front-back, and $2,722 \mathrm{msec}$ for right-left. In the true-false condition, we did find spillover effects into the true-false choice period. Mean choice RTs were $1,181,1,276$, and $1,428 \mathrm{msec}$ for above-below, front-back, and right-left, respectively. The dimension effect for choice times was significant $\left[F(2,56)=6.90, M S_{\mathrm{e}}=\right.$ $67,522]$. In addition, there were significant differences among the RTs to the location words with means of $1,608,1,824$, and $2,299 \mathrm{msec}$ for above-below, front-back, and right-left, respectively $\left[F(2,56)=28.84, M S_{\mathrm{e}}=\right.$ $129,110]$. Because of the spillover effects in the $2 \mathrm{AFC}$ condition, total RTs were formed by summing the location word and choice RTs, and these were used in all analyses. Choice times were quite different in the true-false and the 6 AFC conditions, so total RTs for these two conditions were analyzed separately.

Because there was no single-perspective, other condition in this experiment, the main analyses were from the viewpoint of self. Mean total RTs from the single-perspective, self condition and mean total RTs for the self viewpoint of the multiple-perspective condition were analyzed with 2 (number of perspectives) $\times 3$ (dimension) mixed ANOVAs.

True-false group. Mean RTs for the true-false group are shown in the left half of Figure 3. Again, abovebelow was faster than front-back, which was faster than right-left $\left[F(2,56)=35.65, M S_{\mathrm{e}}=191,117\right.$ for the dimension main effect]. Overall, RTs did not differ significantly in the single- and multiple-perspective conditions $(F<1)$, and number of perspectives did not interact with dimension $(F<1)$. The critical difference bars on the true-false graph in Figure 3 show that above-below judgments were significantly faster than front-back and rightleft judgments, and front-back judgments were faster than right-left judgments.

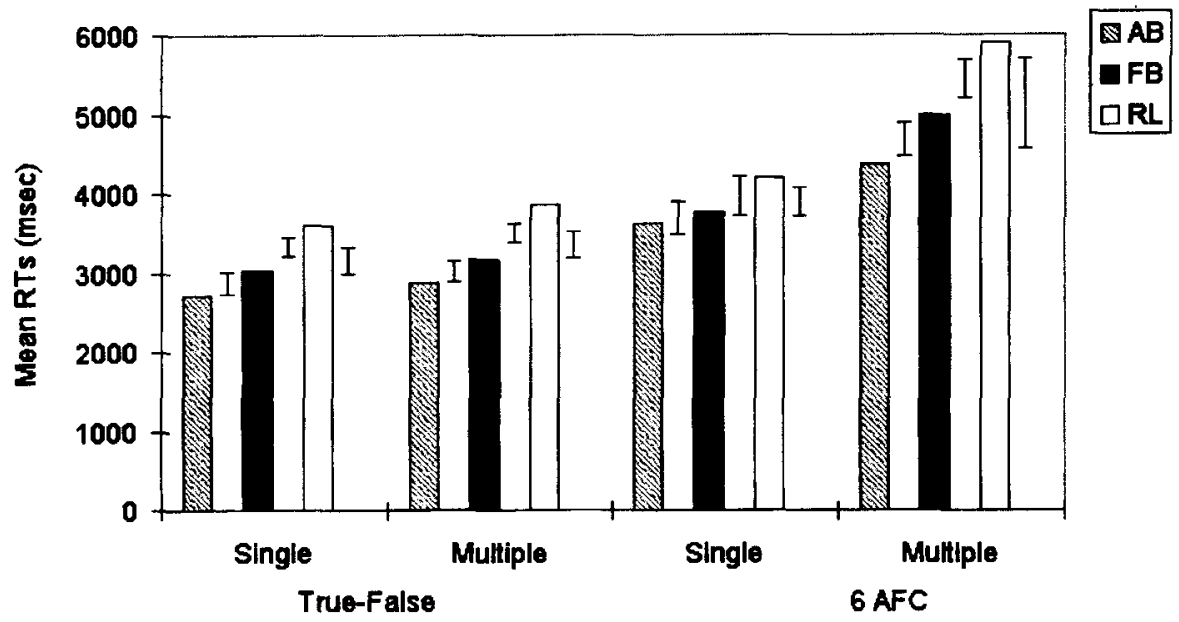

Figure 3. Mean reaction time (RTs) in milliseconds for front-back (FB), above-below (AB), and right-left (RL) locations from the self viewpoint in the single- and multiple-perspective conditions with the true-false and the six-alternative forced-choice procedures of Experiment 3 . Error bars show the Tukey HSDs between above-below and front-back, between front-back and right-left, and between right-left and above-below. 
Proportions correct were $.96, .94$, and .88 for abovebelow, front-back, and right-left, respectively. Responses to above-below and front-back stimuli were accurate about the same proportion of the time, but both dimensions produced more correct responses than did rightleft $\left[F(2,56)=14.31, M S_{e}=.003\right.$, for the dimension main effect]. Overall proportions correct in the single- (.94) and multiple- $(.91)$ perspective conditions were not reliably different $(F<1)$, and dimension and number of perspectives did not interact significantly $[F(1,28)=1.49$, $\left.M S_{\mathrm{e}}=.008\right]$.

Six AFC group. Mean total RTs for the 6 AFC group are shown in the right half of Figure 3 . Unlike the true-false group, the 6 AFC subjects took longer to respond in the multiple-perspective than in the singleperspective condition $\left[F(1,29)=11.59, M S_{\mathrm{e}}=3,059,613\right]$. There was an effect of dimension $\left[F(2,58)=14.72, M S_{\mathrm{e}}=\right.$ $587,704]$, but there was also a trend toward an interaction between dimension and number of perspectives $[F(2,58)=$ $\left.2.95, M S_{\mathrm{e}}=587,704, p=.06\right]$. The interaction trend occurred because the right-left versus above-below comparison interacted with number of perspectives $[F(1,29)=$ 4.64, $\left.M S_{\mathrm{e}}=747,240\right]$. Right-left took longer than above-below when Tukey corrections were applied in both the single- and the multiple-perspective conditions, but the effect was larger in the multiple-perspective condition. Number of perspectives did not interact with the other two pairwise comparisons $\left[F \mathrm{~s}(1,29) \leq 2.05, M S_{\mathrm{e}}=\right.$ $2,346,186]$. Averaged across number of perspectives, the difference between above-below and front-back was not significant with the Tukey correction, although it was without the correction. Right-left took significantly longer than front-back with the Tukey correction.

When the choice involved all six objects, proportions correct were higher in the single- than in the multipleperspective group $\left[.92\right.$ vs. .77; $F(1,29)=9.83, M S_{\mathrm{e}}=$ $.050]$, and proportions correct varied with dimension, .90 , .84 , and .80 , for above-below, front-back, and right-left, respectively $\left[F(2,58)=10.62, M S_{\mathrm{e}}=.007\right]$. There were more correct responses to above-below and front-back than to right-left, and there were more correct responses to above-below than to front-back. Each pairwise comparison was significant with the Tukey correction.

Mixed-viewpoint conditions. Separate analyses were conducted for the true-false and the 6 AFC multipleperspective conditions so that differences from the viewpoint of self and the other person could be investigated. For the true-false group, responding from the viewpoint of self was faster than responding from the viewpoint of other $\left[3,296\right.$ vs. $3,470 \mathrm{msec} ; F(1,13)=5.41, M S_{\mathrm{e}}=$ $117,918]$. In the self condition, mean RTs for above-below, front-back, and right-left were 2,872, 3,162, and $3,853 \mathrm{msec}$, respectively. In the other condition, the corresponding times were $2,837,3,308$, and $4,265 \mathrm{msec}$. Overall, above-below was faster than front-back, and front-back was faster than right-left $[F(2,26)=18.70$, $M S_{\mathrm{e}}=568,054$, for the dimension main effect $]$. Each pairwise comparison was significant with the Tukey cor- rection, but there was no interaction between the two variables $\left[F(2,26)=1.60, M S_{\mathrm{e}}=222,311\right]$.

For the $6 \mathrm{AFC}$ condition, it took somewhat longer to respond from the viewpoint of the other person $(5,325 \mathrm{msec})$ than from the viewpoint of self $(5,091 \mathrm{msec})$, but the difference was not larger than expected by chance $(F<1)$. From the viewpoint of self, mean RTs were 4,380, 4,997, and $5,897 \mathrm{msec}$ for above-below, front-back, and rightleft, respectively. From the viewpoint of the other character, RTs in the same conditions were 4,432, 5,290, and $6,256 \mathrm{msec}$. Again, above-below was faster than frontback, which was faster than right-left $[F(2,28)=10.20$, $M S_{\mathrm{e}}=2,059,802$, for the dimension main effect]. All pairwise comparisons were significant with Tukey corrections, and there was no interaction between viewpoint and dimension $(F<1)$.

Individual differences. In each of our experiments, accuracy rates were lower than those of Franklin et al. (1992). A possible reason for our finding egocentric spatial framework effects is that individuals who are not skilled at spatial judgments may be more likely to use egocentric and intrinsic viewpoints than are individuals who are more skilled. In order to test this hypothesis, we examined subjects who showed high and low accuracy in the $6 \mathrm{AFC}$ task, because this condition was our replication of Franklin et al.'s Experiment 1. The subjects were divided into high and low accuracy on the basis of the median of their condition-single versus multiple perspectives. In the single-perspective condition, the mean accuracy in the high group was .98 and the mean accuracy in the low group was .86. In the multipleperspective condition, the mean high accuracy was .89 and the mean low accuracy was .66. High-accuracy subjects in the multiple-perspective group still showed lower accuracy than did Franklin et al.'s subjects, who scored about .98 correct. However, if we observed spatial framework effects because our subjects were less skilled than Franklin et al.'s, then we should have seen larger spatial framework effects for the low-accuracy than for the highaccuracy subjects in the multiple-perspective condition.

Mean differences obtained by subtracting the abovebelow from the front-back RT, the front-back from the right-left RT, and the above-below from the right-left RT are shown in Figure 4. As can be seen, the size of the spatial framework effects varied across conditions. An ANOVA showed an interaction among dimension, number of perspectives, and level of accuracy $[F(2,54)=$ $\left.3.68, M S_{\mathrm{e}}=524,616\right]$. Thus, we calculated separate critical differences for each of the four accuracy-level by number-of-perspective groups. The critical differences with the Tukey correction are displayed as error bars above zero to the left of each difference. The critical differences are large because each group contained only 6-9 subjects. The multiple-perspective, high-accuracy group is of most interest, because it was the one most similar to Franklin et al.'s (1992) group. For this group, both front-back and right-left took significantly longer than above-below when the Tukey correction was applied. 


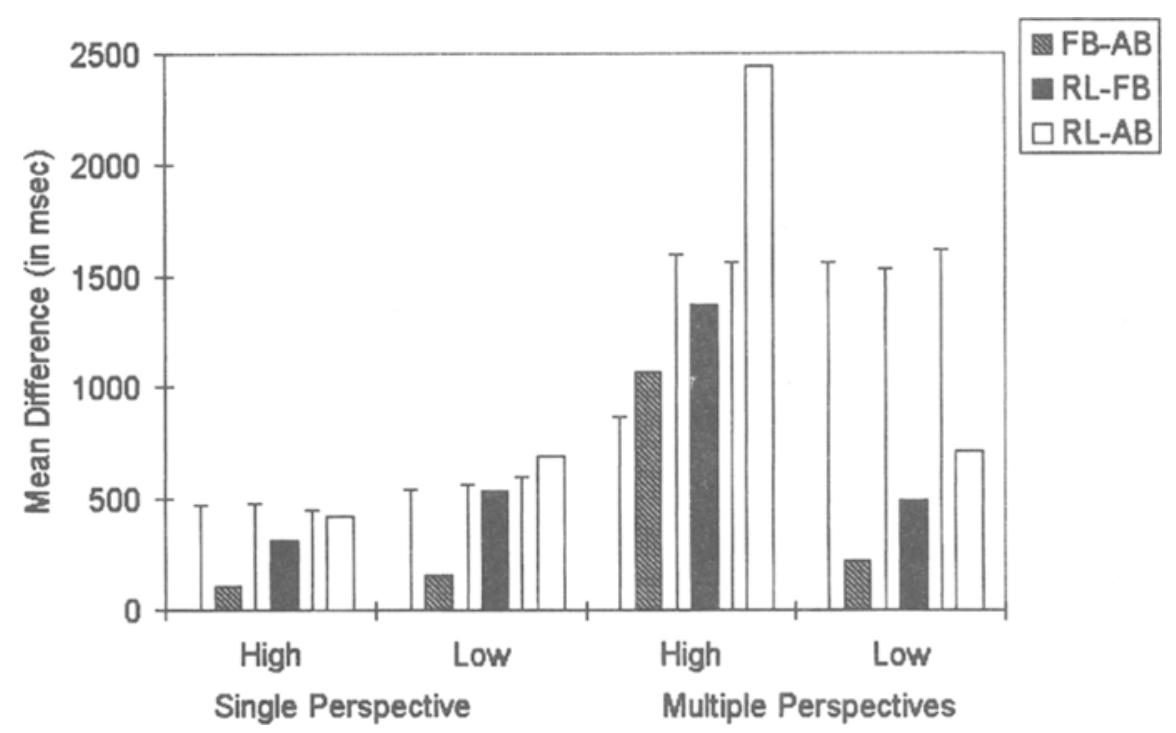

Figure 4. Mean differences in RT (in milliseconds) computed by subtracting above-below from front-back, front-back from right-left, and above-below from right-left for subjects who were high or low in accuracy in the single- and multiple-perspective, six-alternative forced-choice groups in Experiment 3. Error bars show the Tukey HSD, based on the specific data used in each comparison. Significant differences are indicated by data bars that are higher than error bars.

Although right-left also took longer than front-back, this effect was not significant with the Tukey correction, but it was significant without the correction. The other groups showed spatial framework effects that were more variable. For the single-perspective high-accuracy group, the only effect that was significant without the Tukey correction was that right-left took longer than above-below, but this effect was not significant when the correction was applied. For the single-perspective, lowaccuracy group, right-left took significantly longer than above-below with the Tukey correction, and right-left took significantly longer than front-back without the Tukey correction but not with it. There were no significant spatial framework effects for the low-accuracy, multiple-perspective group. Thus, lower accuracy by our subjects cannot explain why we found spatial framework effects in the multiple-perspective condition and Franklin et al. did not.

Practice effects. Another difference between our multiple-perspective condition and that of Franklin et al. (1992) is that we used one practice scenario and four actual scenarios. They used one practice scenario and six actual scenarios. It is possible that spatial framework effects became smaller (or reversed) with practice and that the overall means across their six scenarios eliminated spatial framework effects. We might have seen a similar phenomenon had we continued testing for a total of six scenarios. We examined this by looking at the size of the spatial framework effects across blocks. Our main interest was whether the size of these effects would become progressively smaller with blocks.

In Figure 5, RTs for above--below were subtracted from RTs for front-back, RTs for front-back were subtracted from RTs for right-left, and RTs for above-below were subtracted from RTs associated with right-left for each of the four blocks in the single- and multiple-perspective conditions. The critical difference with the Tukey correction is shown to the left of the bars. Overall, front-back took longer than above-below; this was significant without the Tukey correction, but the difference was not significant when the Tukey correction was applied. There were no clear trends across blocks, as is indicated by the nonsignificant interaction of the front-back versus above-below effect with block $\left[F(3,87)=1.66, M S_{\mathrm{e}}=\right.$ $1,770,137]$ and the nonsignificant three-way interaction with block and perspective group $\left[F(3,87)=1.29, M S_{\mathrm{e}}=\right.$ $1,770,137]$.

The difference between right-left and front-back for each block is shown in Figure 5, with the Tukey critical the difference to the left. Overall, this difference was significantly greater than zero when the Tukey correction was applied. However, this effect interacted with block $\left[F(3,84)=3.16, M S_{e}=1,986,403\right]$. The differences were largest for the first and last blocks, so this is not evidence for a progressive decline in the effect across blocks. The three-way interaction of the right-left versus front-back effect with block and number of perspectives was not significant $\left[F(1,28)=1.60, M S_{\mathrm{e}}=1,986,403\right]$.

An analysis of the right-left versus the above-below difference showed that it was significant overall with the Tukey correction. Although there was some variance in the size of the right-left effect across blocks, it did not interact with block $(F<1)$, and it did not enter into a threeway interaction with block and condition $[F(3,84)=$ $\left.1.97, M S_{\mathrm{e}}=2,431,774\right]$. Differences in the amount of practice probably cannot explain why we found egocentric 


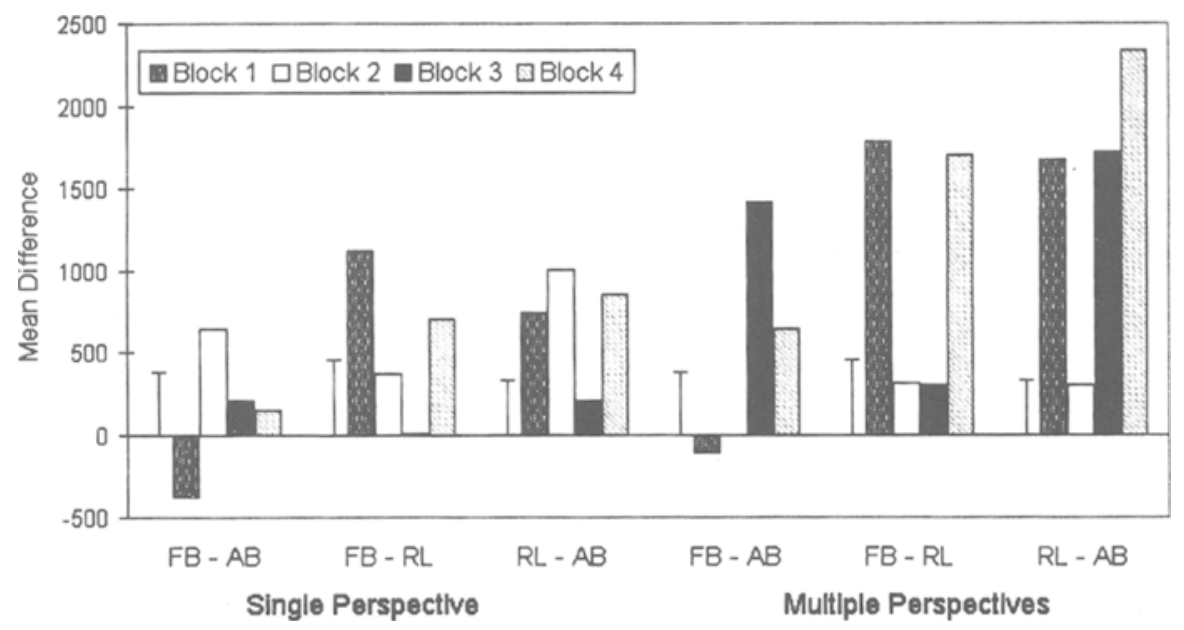

Figure 5. Mean differences in RT (in milliseconds) computed by subtracting above-below from front-back, right-left from front-back, and above-below from right-left for the four blocks in the single- and multiple-perspective, six-alternative forced-choice groups in Experiment 3. Error bars show the Tukey HSD, based on the overall comparison averaged across blocks. Significant differences are indicated by data bars that are higher than error bars.

spatial framework effects in the multiple-perspective condition and Franklin et al. (1992) did not.

Within-dimension analyses. We also analyzed the difference between front and back. In the $6 \mathrm{AFC}$, singleperspective condition, front took $3,591 \mathrm{msec}$ and back took $3,919 \mathrm{msec}$. In the multiple-perspective condition, front took $4,556 \mathrm{msec}$ and back took $5,437 \mathrm{msec}$. A 2 (number of perspectives) $\times 2$ (direction) ANOVA showed that front was faster than back $[F(1,29)=10.95$, $\left.M S_{\mathrm{e}}=516,532\right]$, but this did not interact with number of perspectives $\left[F(1,29)=2.29, M S_{\mathrm{e}}=516,532\right]$. In the true-false condition, however, front produced RTs that were similar to back ( 3,040 vs. $3,152 \mathrm{msec}, F<1)$, and this did not interact with number of perspectives $[F(1,28)=$ $\left.1.61, M S_{\mathrm{e}}=230,904\right]$.

\section{Discussion}

When subjects decided whether an object's location was true or false, we observed clear spatial framework effects in that both front-back and right-left took longer than above-below. When subjects selected from among six objects, the front-back versus above-below comparison was not significant with the Tukey correction, although it was significant without the correction. However, right-left took longer than front-back and above-below in the $6 \mathrm{AFC}$ condition. The multiple-perspective, $6 \mathrm{AFC}$ condition was a replication of Franklin et al.'s (1992) Experiment 1. For questions involving the self perspective, they did not find reliable differences among above--below, front-back, and right-left.

In some ways, we replicated Franklin et al.'s (1992) results in the $6 \mathrm{AFC}$ condition, in that front-back did not take significantly longer than above-below if the Tukey correction was applied (although the difference was signif- icant without the correction). The reduction in the size of the front-back versus above--below effect was not due to taking multiple versus single perspectives, however, because number of perspectives did not interact with the front-back versus above-below effect. Also, front-back took longer than above-below with the true-false procedure, regardless of the number of perspectives. Thus, although the front-back versus above-below effect was reduced in the $6 \mathrm{AFC}$ condition relative to other conditions, the best interpretation is that the reduction in effect size resulted from adding more objects as alternatives during choice rather than requiring responses from more viewpoints.

Our 6 AFC multiple-perspective condition differed from that of Franklin et al. (1992), in that our subjects made more errors than theirs did. However, our more accurate subjects showed more reliable spatial framework effects than did our less accurate subjects, so lower accuracy for our subjects cannot explain why we found reliable spatial framework effects and Franklin et al. did not. Furthermore, the difference between our results and Franklin et al.'s cannot be explained by the fact that our subjects had less practice than did Franklin et al.'s, because there were not clear trends across blocks.

For the $6 \mathrm{AFC}$ data, front was responded to more quickly than back, as it was in the first two experiments. Bryant et al. (1992) argued that this pattern suggests that subjects responded from an egocentric spatial framework. The true-false data showed a similar pattern, but front was not significantly faster than back. Why the number of response alternatives should influence RTs to front versus back is unclear. However, in no case was there evjdence that the requirement to take single or multiple perspectives in a scene produced different patterns. 


\section{GENERAL DISCUSSION}

In almost every case across these experiments, the RTs for front-back judgments were significantly longer than the RTs for above-below judgments with Tukey corrections. Two exceptions were in the single-perspective, self condition of Experiments 1 and 2 and in the 6 AFC condition of Experiment 3. In the first case, the front-back versus above-below comparison was significant in the multiple- but not in the single-perspective condition. In the second case, the front-back, above-below difference was significant without the Tukey correction, but not with the correction. In every condition, however, right-left judgments took longer than both front-back and abovebelow judgments with Tukey corrections. Thus, the rightleft difficulty described by Harris (1975) and by Maki et al. (1977) was replicated with these imagined scenarios. The overall pattern across dimensions suggests the use of egocentric or intrinsic spatial frameworks of the type described by Franklin and Tversky (1990). The fact that the egocentric pattern occurred for both the self and the other character suggests that subjects took each viewpoint in turn and responded from the appropriate character's egocentric viewpoint.

Franklin et al. (1992) proposed a one-place, oneperspective rule. They hypothesized that subjects use a single perspective even when there are two characters having different viewpoints in a common environment. This is accomplished by creating a neutral, external perspective in which spatial locations are equally available. Such a neutral perspective is in contrast to the type of internal, egocentric perspective that is used when there is a single viewpoint. If the one-place, one-perspective rule were operating, dimension effects should have been larger in the single-perspective conditions than in the multiple-perspective condition. The interaction between dimension and number of perspectives did occur in some conditions, but in each case, the size of the dimension effect was larger when responses were made from multiple perspectives as opposed to a single perspective. The finding of larger spatial framework effects in the multipleperspective condition than in the single-perspective conditions supports Schober \& Bloom's (1995) conclusion that it is particularly difficult to take another person's perspective along with one's own perspective. Responding from multiple points of view was particularly difficult for right-left decisions. In most cases in which the multipleperspective condition produced larger effects than did the single-perspective conditions, it was because the time to respond to right and left was particularly long.

Our results are consistent with those of de Vega (1994), who found that right-left took longer than front-back when subjects needed to respond from the perspective of two characters having different orientations. De Vega interpreted his results as showing that subjects held a perspective-free framework in memory which included the objects, "self," and other characters. During the ver- ification items, subjects "fleshed" out this framework to instantiate a particular character's perspective, and to compute the specific spatial relations from that character's perspective. However, de Vega did not examine the most critical comparison for the one-place, one-perspective rule - namely, the comparison of above-below versus front-back. Our results extend de Vega's finding to show that above-below is faster than both front-back and right-left when multiple viewpoints are required. Consistent with de Vega's interpretation of his data, our subjects may have stored the scenarios in a neutral manner, but they took the viewpoint of each character in order to respond to the object location queries computing specific spatial relations during the response time. It was more difficult to compute right--left relations than front-back and above-below relations, and it was more difficult to compute front-back relations than above-below relations.

De Vega (1994) found no asymmetry between front and back in his experiments, so he concluded that subjects stored the locations with the use of an external rather than an internal framework. Franklin et al. (1992) did not report data from within-dimension comparisons, but their hypothesis about a single, external perspective suggests that front should not have been faster than back in their experiments. However, we found that front was faster than back in most of our experiments, whether the viewpoint was self or other. This front versus back effect was never modulated by the need to respond to scenarios from a single versus a multiple perspective. If we accept Bryant et al.'s (1992) suggestion that faster responses to front than back show the use of an internal egocentric framework, this result adds to the evidence that our subjects took the "egocentric" perspectives of the self and the other character as required for each response.

In six experiments, Franklin et al. (1992) found a significant difference between front-back and above-below in only one experiment. This was when there were two different observers in different places. In some of their other conditions, front-back took longer than above-below, but the differences were not significant. In still other conditions, such as their Experiment 1 (from which our scenarios were taken), there were no differences in the front-back versus above-below means for the self viewpoint. Why did we find spatial framework effects when they did not? We showed that the differences in studies cannot be accounted for by level of accuracy or by practice effects. Thus, we consider other possibilities.

Franklin et al.'s (1992) subjects may have used a strategy that our subjects either could not or did not use. Taylor and Tversky (1996) found that subjects were most likely to use intrinsic, viewer-centered frames of reference when describing environments that were traversed with a single path and that had landmarks that were on a single size scale. However, subjects were more likely to use external, absolute frameworks with the terms east, west, north, and south when the environment that they were describing had multiple paths through it and multi- 
ple size scales. Although it might be argued that the subjects in Franklin et al.'s experiments used absolute frameworks because they needed to deal with multiple perspectives, there are two problems with this interpretation of the data. First, we used the same instructions that Franklin et al. did, and we used the same scenarios. Thus, the environments to which students were responding were the same in our and Franklin et al.'s experiments, so there is no obvious reason why different strategies should have been adopted. Second, the use of these absolute compass directions does not mean that spatial framework effects will not occur. Maki et al. (1977) found that north and south were consistently responded to faster than east and west.

Another possible explanation is statistical power. Using the 6 AFC procedure in their experiments, Franklin et al. (1992) may have not had enough power to detect dimension effects. They used between 10 and 12 subjects in their experiments. We had more power in that we always used 16 subjects per group, and we used both single- and multiple-perspective groups in each experiment. Still, power is probably not the entire explanation, because the differences that we observed were much larger than the small differences sometimes observed by Franklin et al.

A better explanation might be in terms of the specific task used by Franklin et al. (1992). Across conditions, the 6 AFC procedure produced the weakest spatial framework effects that we observed. This procedure produced long RTs, high error rates, and much variability in our data. The variability may have made the task insensitive to spatial framework effects, especially the smallest above-below versus front-back difference.

Whatever may be the best explanation for the differences between our studies and those of Franklin et al. (1992), it is clear that we did not observe the pattern predicted by the one-place, one-perspective rule. The multiple-perspective group never showed smaller egocentric spatial framework effects than the single-perspective groups did. However, our results do provide strong support to the egocentric spatial framework described by Franklin and Tversky (1990). This "egocentric" spatial framework pattern occurred whether subjects were responding from the viewpoint of self or other. Our data clearly support the conclusion that humans can most easily discriminate above from below, followed by front from back, with right-left discriminations being particularly difficult.

\section{REFERENCES}

Bryant, D. J., Tversky, B., \& FrankLin, N. (1992). Internal and external spatial frameworks for representing scenes. Journal of Memory \& Language, 31, 74-98.

Carlson-Radvansky, L. A., \& IRwin, D. E. (1994). Reference frame activation during spatial term assignment. Journal of Memory \& Language, 33, 646-671.

Clark, E. V. (1980). Here's the top: Nonlinguistic strategies in the acquisition of orientational terms. Child Development, 51, 329-338.

DE VEGA, M. (1994). Characters and their perspectives in narratives describing spatial environments. Psychological Research, 56, 116-126.

Franklin, N., \& TVERSKY, B. (1990). Searching imagined environments. Journal of Experimental Psychology: General, 119, 63-76.

Franklin, N., TVERSKy, B., \& COON, V. (1992). Switching points of view in spatial mental models. Memory \& Cognition, 20, 507-518.

HARRIS, L. J. (1972). Discrimination of left and right, and development of the logic of relations. Merrill-Palmer Quarterly of Behavior \& Development, 18, 307-320.

HARRIS, L. J. (1975). Spatial direction and grammatical form of instructions affect the solution of spatial problems. Memory \& Cognition, 3, 329-334.

Harris, L. J., \& Strommen, E. A. (1979). The development of understanding of the spatial terms front and back. In H. Reese (Ed.), Advances in child development and behavior (Vol. 14, pp. 149-207). New York: Academic Press.

Hintzman, D., O'Dell, C., \& Arndt, D. (1981). Orientation in cognitive maps. Cognitive Psychology, 13, 149-206.

KEPPEL, G., \& ZEDECK, S. (1989). Data analysis for research designs. New York: W. H. Freeman.

LoGAN, G. D. (1995). Linguistic and conceptual control of visual spatial attention. Cognitive Psychology, 28, 103-174.

MaKi, R. H., Grandy, C., \& HaUGE, G. (1979). Why is telling right from left more difficult than telling above from below? Journal of Experimental Psychology: Human Perception \& Performance, 5, 52-67.

Makı, R. H., Maki, W. S., JR., \& MarSh, L. G. (1977). Processing locational and orientational information. Memory \& Cognition, 5, 602-612.

Rudell, R., \& TEUBER, H. (1963). Discrimination of direction of line in children. Journal of Comparative \& Physiological Psychology, 56, 892-898.

SCHOber, M. F. (1993). Spatial perspective-taking in conversation. Cognition, 47, 1-24.

Schober, M. F. (1995). Speakers, addressees, and frames of reference: Whose effort is minimized in conversations about locations? Discourse Processes, 20, 219-247.

Schober, M. F., \& BLOOM, J. E. (1995, November). The relative ease of producing egocentric, addressee-centered, and object-centered spatial descriptions. Paper presented at the meeting of the Psychonomic Socicty, Los Angeles.

Sholl, M. J., \& EgeTh, H. E. (1981). Right-left confusion in the adult: A verbal labeling effect. Memory \& Cognition, 9, 339-350.

TAYLOR, H. A., \& TVERSKY, B. (1996). Perspective in spatial descriptions. Journal of Memory \& Language. 35, 371-391.

(Manuscript received February 23, 1996; revision accepted for publication September 19, 1996.) 